\title{
Les transports publics à l'épreuve des mutations de la pendularité
}

\author{
Comparaisons diachroniques sur la base des résultats \\ des recensements fédéraux de I97o, Ig8o, I99o et 2 ooo.
}

\section{Martin Schuler et Vincent Kaufmann}

Martin Schuler est professeur au Laboratoire Chôros de l'Ecole polytechnique fédérale de Lausanne, Suisse.

Vincent Kaufmann est professeur au Laboratoire de sociologie urbaine de l'Ecole polytechnique fédérale de Lausanne, Suisse.
Since 1970 , the Swiss Census has enabled a picture of the working population's commuting pattern to be drawn up periodically. Available data covers not only the route between home and workplace, but also the frequency, time expenditure and mode of transportation of these trips. This article specifically deals with the topic of long-distance commutes between some of the country's major cities: Zurich and Bern, Geneva and Lausanne. This type of flow has increased strongly, though it is still very clearly oriented towards public transportation. While it is true that the proportion of train commuters has fallen, this is due to an increase in the number of connections to and from the outer rings of cities, where car-users have a clear time advantage, and to the relative loss of the share of inter-city commuters.

For Switzerland as a whole, the r97os were a period of motorisation and decreasing public transportation use. The i 98 os brought a return of the latter trend because of significant improvements made to the railway system and because the period was characterized by a growing awareness of environmental issues. Between I990 and 2000, road transport expanded constantly and the use of the train for commuting increased essentially for long-distance trips and within the Greater Zurich Area, where a very efficient urban transportation network has been built. In an international comparison, Switzerland has not only developed an efficient public transportation system, it has also maintained a relatively high proportion of railway and urban transport system users.

\section{Introduction}

La promotion de l'utilisation des transports publics pour aller travailler est depuis une vingtaine d'années une priorité en matière de politique des transports en Suisse. Pourtant, après les résultats encourageants observés durant la décennie r $980-$ I $99^{\circ}$, force est de constater que l'utilisation de l'automobile pour aller travailler continue de croître, ceci malgré des investisse- ments parfois considérables dans les infrastructures de transports publics urbains (construction de tramways) ou régionaux (S-Bahn-Netze). Le présent article propose d'analyser ces évolutions à la lumière des mutations qu'a connues la pendularité ces dernières années afin de dégager les conditions dans lesquelles les transports publics peuvent rester compétitifs par rapport à l'automobile. Pour ce faire, une analyse diachronique (I 970, I980, r 990,2000 ) de la mobilité pendulaire ${ }^{1}$ des personnes actives est réalisée dans les agglomérations suisses. Il ressort de ce travail un certain nombre d'observations de nature à contribuer au débat sur le péage urbain, dont l'acceptabilité politique est actuellement très faible en Suisse (ARE 200I).

\section{Problématique}

En Europe, depuis environ une décennie, la mobilité de la vie quotidienne est en mutation. Plus précisément, deux tendances se font jour: l'accroissement des budgets-temps consacrés quotidiennement à se déplacer et l'émergence de nouvelles formes de mobilité.

\section{I L'accroissement des budgets-temps de déplacement}

Formulée sur la base de multiples analyses et vérifiée dans plusieurs cas, la «conjecture de Zahavi» postule que l'ensemble des temps de déplacements reste stable au fil des années, et que les gains de temps obtenus par l'amélioration des infrastructures (autoroutes, contournements routiers, accroissement de l'offre de transport public, améliorations techniques des moyens de transport) ne sont pas utilisés par l'individu pour mener d'autres activités mais sont au contraire réinvestis dans les déplacements d'où l'augmentation des distances parcourues. Une personne voyant son temps de trajet réduit de 20 minutes au fil des ans préférerait ainsi s'éloigner de 20 minutes supplémentaires de son lieu de travail, en s'installant dans le périurbain, plutôt que de consacrer ces 
20 minutes supplémentaires aux activités familiales, par exemple. L'économie de temps est dépensée par l'individu dans l'élargissement des potentialités de choix de sa résidence, des lieux de travail, d'achat ou de loisir.

Des enquêtes récentes mettent en évidence une croissance du temps consacré aux déplacements dans la vie quotidienne. La conjecture de Zahavi semble donc infirmée: le budget-temps consacré en movenne à se déplacer par personne et par jour n'est plus stable, mais il augmente, tout comme le nombre de déplacements effectué en moyenne par chaque citoyen. Le pendant de cette situation est un accroissement considérable des prestations de trafic automobile, avec les conséquences que cela implique en termes environnementaux, de consommation d'énergie et de congestion. En Suisse par exemple, selon les chiffres des deux derniers micro-recensements transport, le budget-temps consacré quotidiennement aux déplacements est passé de 84 minutes en r 994 à 94 minutes en 2000.

Même si la recherche est encore balbutiante sur l'accroissement des budgets-temps consacrés aux déplacements, des facteurs susceptibles d'être à l'origine de cette tendance sont identifiés:

Nous assistons tout d'abord à un découplage entre la vitesse de transport et la mobilité. L'enquête menée par Crozet et Joly (2003) a montré que le mécanisme de compensation de l'accroissement des kilomètres parcourus par les gains de vitesse ne fonctionne plus. Ainsi par exemple, la comparaison des micro-recensements indique que globalement les déplacements en automobile sont presque $4 \mathrm{~km} / \mathrm{h}$ plus lents en 2000 qu'en r 994 (passant de 42,2 km/h à 38,6 $\mathrm{km} / \mathrm{h}$ ), alors que dans le même temps le nombre moyen de kilomètres parcourus augmentait de $2,5 \mathrm{~km}$ par jour en moyenne. Les personnes qui se déplacent le plus loin sont désormais aussi celles qui ont les budgets-temps consacrés aux déplacements les plus importants. Des changements économiques et sociétaux contribuent à expliquer cette tendance: le changement de la répartition spatiale des emplois et des $\log \mathrm{e}-$ ments au profit des couronnes d'agglomération, l'élargissement des bassins de vie, qui génèrent des déplacements professionnels d'une portée spatiale plus étendue, la précarisation du travail qui oblige parfois à accepter des temps de déplacements très importants, les arbitrages de localisation résidentielle des couples de bi-actifs.

Le rapport au temps de déplacement est en train de changer. De temps de liaison difficilement utilisables, ils deviennent des temps sociaux à part entière. Le déplacement de loisir en voiture est un moment privilégié du cvivre ensemble» familial. Le train pour des déplacements pendulaires de longue distance est de plus en plus utilisé comme lieu de travail (grâce notamment à la complicité du téléphone mobile et de l'ordinateur portable). Le chaînage des activités se construit par une appropriation des espaces traversés (s'arrêter en passant dans un supermarché, faire un crochet pour chercher un enfant à un cours, etc.). Dans ce contexte, des budgets-temps de déplacement plus élevés sont tolérés car l'utilité des temps de mobilité s'est améliorée.

L'accroissement des budgets-temps est aussi une conséquence des politiques de transports urbains menés dans les grandes agglomérations. Une recherche comparative récente sur les inégalités sociales d'accès dans des agglomérations suisses et françaises le montre bien (Flamm 2004): les agglomérations dans lesquelles une politique de réduction des usages urbains de l'automobile a été menée dans les années r $99^{\circ}$ (Zurich, Berne) se caractérisent par des reports modaux de l'automobile vers les transports publics entraînant des accroissement des budgets-temps.

\section{I L'émergence de formes transversales de mobilité}

Avec la diffusion des technologies de l'information et de la communication, la pratique de chacune de ces mobilités change au point que celles-ci se brouillent. Les distances et les vitesses croissent, tandis que les temporalités auxquelles elles renvoient se désynchronisent pour se re-synchroniser selon de nouvelles modalités. Si ce phénomène semble assez général en Europe, il prend en Suisse la forme d'une mise en réseau des villes qui constitue un changement important de la structure urbaine: il est de moins en moins rare de travailler dans une autre agglomération que celle dans laquelle on réside. Cela se traduit en particulier par l'émergence de deux nouvelles formes de mobilité:

- La bi-résidentialité. Si le double domicile saisonnier existe depuis longtemps, en revanche son développement à l'échelle de la semaine est récent. Il recouvre des situations très différentes. Parmi les couples de bi-actifs, il est souvent le résultat d'arbitrages familiaux lorsque les activités professionnelles des conjoints ne sont pas localisées dans la même aggolomération (Lévy 200o). On assiste également depuis quelques années au développement de pratiques de double domicile avec les résidences secondaires habitées trois jours par semaine. 
- Lorsque le lieu de travail est très éloigné du domicile, une autre pratique que le double domicile se développe actuellement: la pendularité de longue distance associée au travail à domicile. Elle consiste à ne se déplacer sur son lieu de travail que deux ou trois jours par semaine et à travailler le reste du temps à son domicile (Viard I 995). Cette pratique s'appuie largement sur les possibilités de travailler à distance procurées par la messagerie électronique.

Si ces nouvelles formes de mobilité ne représentent encore qu'un petit pourcentage des actifs, notons que celui-ci est en forte progression.

Ces deux formes de mobilité ont en commun de concerner le travail et de se fonder largement sur l'usage des technologies de l'information et de la communication. Elles jouent de fait sur la combinaison des vitesses entre transport et systèmes de communication et les capacités des acteurs à s'approprier les systèmes techniques. Elles consistent souvent à renoncer à développer des formes de mouvement et permettent d'éviter de choisir entre des alternatives comme déménager ou pas pour prendre un nouvel emploi, être en couple ou vivre seul, etc. Mentionnons enfin qu'elles sont de nature à favoriser l'allongement des budgets-temps consacrés aux déplacements.

En fin de compte, l'allongement des budgets-temps et le développement de nouvelles formes de mobilité suggèrent que la conjecture de Zahavi n'est peut être qu'un hasard de circonstances, lié à trois choses:

- Des phénomènes de co-variation. La croissance de certaines formes de mobilités (les loisirs en voiture, la pendularité de longue distance en voiture ou en train) a longtemps été compensée par la diminution d'autres formes de mobilité (le retour à midi, les déplacements à pied, qui représentent vite beaucoup de minutes, mais peu de kilomètres);

- La non-appropriation des temps de déplacements. L'appropriation de plus en plus fréquente des temps de déplacements par les usagers, favorisée par l'amélioration du confort et par des innovations techniques rend acceptables des budgets-temps de déplacement plus longs (Flamm 2004);

- Des durées de déplacements quotidiens qui sont limitées par un temps discrétionnaire restreint. Après déduction des temps physiologiques (sommeil, etc.) et des temps contraints (travail, études, etc.), le temps libre est proche en moyenne de 4 à $5 \mathrm{~h}(4 \mathrm{~h} 30$ en France entre r986-i 999 d'après les enquêtes emploi du temps). Il paraît alors raisonnable, en moyenne de consacrer un quart de ce temps aux déplacements, soit près d'une heure.

Si les deux tendances que nous venons d'évoquer sont générales en Europe, une troisième observation, plus spécifique à la Suisse, peut être faite concernant l'utilisation de l'automobile.

Durant les deux dernières décennies, des investissements considérables ont été faits dans le domaine des transports en Suisse. S'y ajoutent des améliorations notables des fréquences et des mesures tarifaires des transports publics dans les agglomérations. Au plan de l'offre "grande ligne», le programme Rail+Bus 2000 est mis en service par étapes et permet une amélioration importante de la qualité de l'offre ferroviaire à travers tout le pays. Le développement de ces offres nouvelles a largement été pensé pour susciter un report modal de l'utilisation de l'automobile vers celle des transports publics. Or, globalement la fréquentation des transports publics pour les déplacements domicile-travail stagne depuis une dizaine d'années.

Si le recensement de r $99^{\circ}$ avait permis de mettre en relief une progression de l'utilisation des transports publics pour aller travailler par rapport au recensement précédent de i 980 , cette tendance ne s'est pas poursuivie en 2000. Globalement, entre r $99^{\circ}$ et 2000 , l'utilisation de l'automobile progresse au détriment des transports publics, mais cette observation est à nuancer en fonction du type de transports public et de l'agglomération considérée:

- D'une manière générale la chute est particulièrement marquée pour les transports publics urbains (bus, trams) et contenue pour les chemins de fer;

- Des différences importantes sont à relever entre agglomérations. Ainsi, les agglomérations romandes souffrent des baisses les plus marquées, tandis que dans certaines agglomérations alémaniques, dont font parties Zurich, Berne et Bâle, la part des transports publics se maintient.

Le tableau i donne un survol sur l'évolution de la relation entre le taux d'utilisation de l'automobile et des transports publics pour les déplacements pendulaires effectués par l'un de ces deux moyens de transport pour la période entre I 970-200o, ventilé par type de commune; les deux cartes montrent le même indicateur pour les années I 970 et 2000 , mais à l'échelle des régions de mobilité spatiale (MS). L'indicateur utilisé réfère le pourcentage des personnes se rendant en transports publics au travail à celui des personnes qui utilisent la voiture; il ne tient pas compte des personnes qui se déplacent avec des modes de transport non moto- 


\begin{tabular}{lccccccc}
\hline Type de commune & 1970 & 1980 & 1990 & 2000 & $1970-1980$ & $1980-1990$ & $1990-2000$ \\
\hline Centrales & 0,957 & 0,879 & 1,020 & 0,916 & $-0,078$ & 0,141 & $-0,104$ \\
Suburbaines d'emploi & 0,561 & 0,498 & 0,624 & 0,566 & $-0,063$ & 0,126 & $-0,058$ \\
Suburbaines résidentielles & 0,466 & 0,362 & 0,423 & 0,363 & $-0,104$ & 0,061 & $-0,061$ \\
Riches & 0,450 & 0,424 & 0,540 & 0,453 & $-0,026$ & 0,116 & $-0,087$ \\
Périurbaines & 0,372 & 0,263 & 0,329 & 0,278 & $-0,109$ & 0,066 & $-0,051$ \\
Touristiques & 0,278 & 0,201 & 0,231 & 0,187 & $-0,077$ & 0,030 & $-0,044$ \\
Industrielles & 0,402 & 0,237 & 0,251 & 0,200 & $-0,165$ & 0,015 & $-0,051$ \\
Pendulaires & 0,333 & 0,212 & 0,242 & 0,186 & $-0,121$ & 0,030 & $-0,056$ \\
Mixtes & 0,331 & 0,201 & 0,219 & 0,161 & $-0,130$ & 0,018 & $-0,058$ \\
Agricoles & 0,285 & 0,175 & 0,195 & 0,127 & $-0,110$ & 0,019 & $-0,067$ \\
CH & 0,609 & 0,480 & 0,538 & 0,445 & $-0,129$ & 0,058 & $-0,093$ \\
\hline
\end{tabular}

risés (marche, vélo, etc.). Les colonnes des périodes intercensitaires présentent la différence des taux entre le début et la fin de la décennie, indépendamment du niveau.

Ces illustrations mettent en avant, d'une part, la succession très inégale des trois périodes et, d'autre part, la grande différence entre les types de communes: seule dans les centres, la relation entre les deux modes reste équilibré (indice proche de I), tandis que, comme exemple extrême, dans les communes agricoles seuls 0,127 trajets en transports publics sont comptés pour un trajet en voiture. Les écarts entre les types de communes (et également entre les régions) se sont creusés fortement au cours des années r970 et r980; depuis r99o, les villes connaissent également une diminution de l'utilisation des transports publics.

$\mathrm{Si}$, en r 970 , quelques régions périphériques industrielles connaissaient encore une part du marché important des trains et bus, la carte de l'an 2000 montre un faible demande de ceux-ci dans l'ensemble des régions rurales et de petits centres. Après r $99^{\circ}$, l'utilisation des bus urbains et régionaux a baissé, tandis que le train a pu se maintenir, mais ceci uniquement dans des segments très spécifiques.

La stagnation de la fréquentation des transports publics pour les déplacements domiciletravail observée pour l'ensemble de la Suisse va être nuancée pour la pendularité à grande distance. L'analyse des données observées entre quelques grandes agglomérations devra permettre de répondre aux questions de recherches suivantes:

- Les changements observés sont-ils le reflet de l'évolution des durées comparées de déplacements entre domicile et lieu de travail en au- tomobile et en transports publics? En d'autres mots, dans quelle mesure la baisse de part modale des transports publics est-elle liée à la spatialisation des dynamiques de croissance urbaine et d'accessibilité des agglomérations, qui font que lieux de travail et de résidence sont de plus en décentralisés?

- Les changements observés sont-ils le reflet d'une nouvelle attitude à l'égard des temps de déplacement? En d'autres mots, dans quelle mesure la baisse de la part modale des transports publics urbains est-elle la conséquence de l'amélioration qualitative des temps de déplacements en automobile?

- Le maintien de la part du chemin de fer, voire sa croissance est-elle le reflet de l'émergence de nouveaux usages des temps de déplacements? En d'autres mots, les usagers ne seraient-ils plus sensibles à la qualité comparée des temps de déplacement, plutôt que réactifs à la comparaison des durées?

\section{Méthodologie}

Pour répondre à ces questions, nous avons mené des analyses diachroniques sur la base des recensements i970, I980, г990 et 2000. Celles-ci ont été menées pour les cinq grandes agglomérations du pays, Bâle, Berne, Genève, Lausanne et Zurich. Ces agglomérations présentent l'avantage d'être doublement différenciées:

- Au niveau des infrastructures de transport: Zurich, Bâle et Berne ont désormais un réseau de S-Bahn qui a permis de considérablement améliorer la qualité des temps de déplacements en transports publics et l'efficacité de ce moyen
Tableau ı: Relation entre le taux d'utilisation des transports publics et de la voiture, Suisse évolution entre r 970 et 2000. 
de transport (trajets plus directs et meilleures fréquences); pour Lausanne, mentionnons la réalisation du TSOL; Genève dispose d'une autoroute de contournement depuis r 993 , autoroute qui a rendu l'automobile nettement plus concurrentielle par rapport aux transports publics.

- Au niveau de la dynamique urbaine des localisations, Bâle et Berne, et d'un moindre manière Zurich se caractérisent par le maintien d'une certaine compacité, en particulier au niveau de la localisation des emplois, alors que les deux agglomérations romandes se caractérisent par un étalement urbain marqué (soutenu par l'effet de surfaces communales restreintes).

Néanmoins, dans les cinq agglomérations, la dynamique démographique a été nettement plus forte dans les couronnes sub- et périurbaines que dans la ville-centre (seule la ville de Genève a connu une expansion remarquable), et la croissance des emplois a également été plus favorable aux communes suburbaines qu'aux centres. Ainsi, la part des flux pendulaires tangentiels est toujours plus nombreuse et ce sont ceux-ci pour lesquels l'organisation des transports publics est désavantagée.

\section{Analyses}

\section{I Tendances nationales}

La recherche menée par Infras (Frick et al. 2004) sur mandat de l'Office fédéral de la statistique permet une approche générale des évolutions récentes de la pendularité à l'échelle nationale: - L'utilisation des transports privés motorisés pour les trajets entre domicile et travail (et probablement souvent combinés avec d'autres trajets) a fortement augmenté depuis r $99^{\circ}$ et représente en $2000,58 \%$ des trajets pendulaires. Cette augmentation touche l'ensemble du pays, plus encore l'espace rural que les agglomérations.

- La part du train a également augmenté (de 9,8 à I I \%), mais ceci essentiellement dans la métropole zurichoise définie par le périmètre de la S-Bahn, opérationnelle depuis r $99^{\circ}$. En dehors de la région zurichoise, l'utilisation du train a surtout fortement augmenté pour les trajets à longue distance.

- Les transports publics urbains et les bus régionaux perdent des parts de marché (entre I $99^{\circ}$ et 2000 de I 2,3 à I $0,3 \%$ ), étant donné que la clientèle habitant et travaillant dans les villescentres, diminue en chiffres relatifs et absolus.

- Sur l'ensemble de la Suisse, les temps de dé- placements consacrés à la pendularité restent plus ou moins stables. Les résultats sont cependant différents selon l'entité de référence choisie: pour tous les actifs ne travaillant pas à domicile, la durée moyenne du trajet domicile-travail a augmenté d'une minute depuis r 980 (de i 8,3 à 19,4 minutes en 1990 et à I 9,3 en 2000 ); une plus forte croissance du temps moyen est enregistrée si l'on considère tous les actifs (y inclus ceux - toujours moins nombreux - qui n'ont pas de trajet). Par contre, en appliquant le calcul sur la durée moyenne par jour des pendulaires (le temps total consacré par jour aux déplacements pendulaires), la durée moyenne est en régression, puisque le nombre de ces trajets diminue au bénéfice de la disparition progressive des retours à midi.

- En termes de moyens de transports, relevons que la durée moyenne d'un trajet est en baisse pour tous les types de moyens de transport, à l'exception du train (pour lequel elle a passé de 39,4 en r 980 à 42,5 minutes en 2000 ). Nous pouvons en déduire que les vitesses ont fortement augmenté, et donc également les distances parcourues.

- La concentration de la création d'emplois dans les grandes agglomérations s'est accentuée, surtout à partir de la reprise économique de 1995 , ceci au détriment des agglomérations moyennes et de l'espace rural. Au sein des grandes agglomérations, les communes suburbaines accueillent le plus de nouveaux emplois - facilement accessibles par le réseau autoroutier inter-agglomération et les rocades depuis les arrières-pays et les agglomérations voisines.

Par rapport à nos hypothèses, cette première lecture globale indique d'emblée que les tendances observées pour l'ensemble de la mobilité quotidienne ne se vérifient pas pour les déplacements pendulaires. Ainsi les temps de déplacements pendulaires restent stables et les vitesses moyennes par mode de transport augmentent, là où les tendances d'ensemble pour la mobilité globale quotidienne indiquent une croissance des budgets-temps et une diminution des vitesses. Un examen trop rapide de ces divergences pourrait amener à les interpréter comme contradictoires. Ils se complètent pourtant et traduisent des changements en profondeur dans l'organisation de la mobilité.

Les déplacements pendulaires sur lesquels nous travaillons représentent un pourcentage déclinant de la mobilité quotidienne. Pourtant, cette forme de mobilité est de plus en plus structurante de l'ensemble des déplacements de la vie quotidienne car le chaînage des acti- 
vités se développe, et nombreux sont les actifs qui réalisent désormais des boucles de mobilité plus complexes (Kaufmann et al. 20oo), ce qui explique l'apparente contradiction. Le déplacement pendulaire devient de facto le pivot de la mobilité quotidienne des actifs, pivot autour duquel s'organisent les autres activités par enchaînement. Les déplacements pendulaires sont donc au cour d'un paradoxe: leur poids dans l'ensemble de la mobilité quotidienne est faible, mais leur rôle dans les pratiques modales et spatiales est crucial pour l'ensemble des déplacements de la vie quotidienne et pour l'organisation spatiale.

Après ces quelques tendances nationales, intéressons-nous à présent à la pendularité de longue distance.

\subsection{La pendularité inter-agglomération de longue distance}

Avant de traiter des échanges entre agglomérations, intéressons-nous à la pendularité interne aux cinq grandes agglomérations en 2000. La part des transports publics (y compris les trajets multimodaux) sur l'ensemble des déplacements motorisés, est de loin la plus élevée pour les pendulaires habitant et travaillant le centre-ville (Zurich, Bâle et Berne entre 77 et $74 \%$, Genève et Lausanne $57 \%$ ). Par contre, les pourcentages des transports publics sont d'environ 20 points de pour-cent inférieurs pour les trajets allant des communes de couronne vers les centres et de 25 dans le sens inverse. Les flux entre communes de couronne ne s'effectuent guère plus de $20 \%$ par transports publics (avec un maximum de $27 \%$ à Bâle et un minimum de $13 \%$ à Lausanne). La structure des réseaux des transports publics, leur qualité, leur rapidité et les fréquences observées, la disponibilité de parkings à proximité du lieu de travail, mais également des facteurs sociaux régissant l'accès à la voiture sont les facteurs explicatifs clé de ces différences.

Les trajets pendulaires entre les grandes agglomérations ont connu des taux de croissance élevés d'ordre d'un doublement ou d'un triplement par décennie. En règle générale, l'agglomération la plus grande attire des flux plus importants que dans le sens inverse. Ceci s'explique par la concentration des emplois plus spécialisés dans les plus grands centres, les niveaux de salaire et les loyers plus élevés.

La répartition modale pour les trajets interagglomérations est plus favorable aux transports publics pour les liaisons entre deux centres-villes que pour les trajets allant d'une couronne d'agglomération vers une autre couronne d'ag$\operatorname{disP}{ }_{1} 6$ I $\cdot 2 / 2005$ glomération. Partant de ces constats, les flux observés entre les différentes agglomérations se distinguent selon la distance entre celle-ci, la qualité de l'accessibilité et le contexte culturel. L'aspect le plus intéressant est pourtant le changement observé au cours des années r $99^{\circ}$ par rapport à la tendance antérieure. La discussion détaillée s'appuie sur les deux paires d'agglomération de “Zurich-Berne» et de "Genève-Lausanne» (cf. les tableaux 2a à 2d). Ces quatre tableaux distinguent entre la direction des flux et leur orientation sur les centres ou les couronnes et contiennent six indicateurs: une estimation des durées de trajets en train et en voiture et le ratio entre les deux (en tenant compte d'un trajet “moyen» du domicile vers la gare de départ et du trajet depuis la gare d'arrivée jusqu'au site de travail $)^{2}$; le nombre de pendulaires en chiffres absolus et la part modale du train en pourcent. Le dernier indicateur montre la décomposition des flux entre agglomérations par rapport à la distinction entre centre et couronne.

\subsection{Les relations Berne-Zurich (et vice versa)}

En 2000 , environ $75 \%$ des trajets se font en train dans les deux sens. La part du train a même augmenté entre r 990 et 2000 ; tandis qu'au cours des années r 970, elle avait clairement diminué et était restée stable durant les années i980. Si les transports publics sont absolument dominants pour la relation «ville-ville» $(92 \%)$, les relations "ville-couronne» et "couronne-ville» atteignent également entre 70 et $75 \%$. Seule la relation "couronne-couronne» n'est orientée qu'à 36 et $39 \%$ sur le train. Le phénomène remarquable est le fait que le train ait pu récupérer des parts modales pour toutes les liaisons observées, et ceci notamment au cours de la dernière décennie. Sur l'ensemble des trajets, le train a gagné, entre r 990 et 2000 de $66 \%$ à $75 \%$ entre Berne et Zurich et de $6 \mathrm{I} \%$ à $74 \%$ dans le sens inverse. Entre i 98 o et I $99^{\circ}$, la part du train sur l'ensemble des trajets était restée stable, malgré une amélioration sur les quatre types de liaisons - sous l'effet de la forte croissance des trajets “couronne-couronne». Les années I $99^{\circ}$ ont amené une recomposition de ces flux en faveur des trajets entre les villes-centres. En effet, si, en r99o, seule $30 \%$ des trajets entre les agglomérations de Berne et de Zurich (et $34 \%$ dans l'autre sens) se faisaient entre les deux villes, ces pourcentages ont augmenté à $37 \%$ (et $40 \%$ respectivement). Auparavant, de i 970 à I $99^{\circ}$, le poids relatif des flux inter-villes avaient constamment diminué. 


\begin{tabular}{|c|c|c|c|c|c|c|}
\hline $\begin{array}{l}\text { de BERNE } \\
\text { à ZURICH }\end{array}$ & $\begin{array}{l}\text { train } \\
(\text { min. })\end{array}$ & $\begin{array}{l}\text { route } \\
\text { (min.) }\end{array}$ & $\begin{array}{l}\text { rel. de } \\
\text { durée }\end{array}$ & $\begin{array}{l}\text { pendulaires } \\
\text { (chiff. absolus) }\end{array}$ & $\begin{array}{l}\text { \% transports } \\
\text { publics }\end{array}$ & $\begin{array}{l}\text { part du flux } \\
\text { total }\end{array}$ \\
\hline ville/ville 70 & 129 & 123 & 1,05 & 31 & 81 & 49,2 \\
\hline ville/ville 80 & 122 & 100 & 1,22 & 90 & 78 & 33,6 \\
\hline ville/ville 90 & 112 & 100 & 1,12 & 224 & 78 & 30,5 \\
\hline ville/ville 00 & 112 & 100 & 1,12 & 499 & 92 & 37,2 \\
\hline ville/couronne 70 & 152 & 128 & 1,19 & 3 & & 4,8 \\
\hline ville/couronne 80 & 145 & 105 & 1,38 & 34 & 50 & 12,7 \\
\hline ville/couronne 90 & 126 & 99 & 1,27 & 114 & 65 & 15,5 \\
\hline ville/couronne 00 & 120 & 99 & 1,21 & 181 & 75 & 13,5 \\
\hline couronne/ville 70 & 146 & 115 & 1,27 & 26 & 69 & 41,3 \\
\hline couronne/ville 80 & 144 & 97 & 1,48 & 106 & 67 & 39,6 \\
\hline couronne/ville 90 & 125 & 97 & 1,29 & 211 & 77 & 28,7 \\
\hline couronne/ville 00 & 125 & 97 & 1,29 & 386 & 78 & 28,8 \\
\hline couronne/couronne 70 & 169 & 120 & 1,41 & 3 & & 4,8 \\
\hline couronne/couronne 80 & 167 & 102 & 1,64 & 38 & 26 & 14,2 \\
\hline couronne/couronne 90 & 139 & 96 & 1,45 & 196 & 35 & 26,7 \\
\hline couronne/couronne 00 & 133 & 96 & 1,39 & 276 & 39 & 20,6 \\
\hline agglo/agglo 70 & & & & 63 & 73 & 100,0 \\
\hline agglo/agglo 80 & & & & 268 & 63 & 100,0 \\
\hline agglo/agglo 90 & & & & 734 & 66 & 100,0 \\
\hline agglo/agglo 00 & & & & 1342 & 75 & 100,0 \\
\hline
\end{tabular}

\begin{tabular}{|c|c|c|c|c|c|c|}
\hline $\begin{array}{l}\text { de ZURICH } \\
\text { à BERNE }\end{array}$ & $\begin{array}{l}\text { train } \\
(\text { min. })\end{array}$ & $\begin{array}{l}\text { route } \\
(\mathrm{min} .)\end{array}$ & $\begin{array}{l}\text { rel. de } \\
\text { durée }\end{array}$ & $\begin{array}{l}\text { pendulaires } \\
\text { (chiff. absolus) }\end{array}$ & $\begin{array}{l}\text { \% transports } \\
\text { publics }\end{array}$ & $\begin{array}{l}\text { part du flux } \\
\text { total }\end{array}$ \\
\hline ville/ville 70 & 128 & 128 & 1,00 & 26 & 81 & 76,5 \\
\hline ville/ville 80 & 121 & 103 & 1,17 & 65 & 74 & 44,2 \\
\hline ville/ville 90 & 111 & 103 & 1,08 & 128 & 84 & 33,9 \\
\hline ville/ville 00 & 111 & 103 & 1,08 & 385 & 91 & 40,1 \\
\hline ville/couronne 70 & 148 & 121 & 1,22 & 3 & & 8,8 \\
\hline ville/couronne 80 & 145 & 103 & 1,41 & 6 & 67 & 4,1 \\
\hline ville/couronne 90 & 126 & 97 & 1,30 & 28 & 50 & 7,4 \\
\hline ville/couronne 00 & 126 & 97 & 1,30 & 65 & 68 & 6,8 \\
\hline couronne/ville 70 & 149 & 125 & 1,19 & 4 & & 11,8 \\
\hline couronne/ville 80 & 142 & 103 & 1,38 & 67 & 51 & 45,6 \\
\hline couronne/ville 90 & 123 & 103 & 1,19 & 152 & 56 & 40,2 \\
\hline couronne/ville 00 & 120 & 103 & 1,17 & 381 & 69 & 39,7 \\
\hline couronne/couronne 70 & 169 & 121 & 1,40 & 1 & & 2,9 \\
\hline couronne/couronne 80 & 166 & 103 & 1,61 & 9 & & 6,1 \\
\hline couronne/couronne 90 & 138 & 97 & 1,42 & 75 & 36 & 19,8 \\
\hline couronne/couronne 00 & 132 & 97 & 1,36 & 129 & 36 & 13,4 \\
\hline agglo/agglo 70 & & & & 34 & 76 & 100,0 \\
\hline agglo/agglo 80 & & & & 147 & 61 & 100,0 \\
\hline agglo/agglo 90 & & & & 378 & 61 & 100,0 \\
\hline agglo/agglo 00 & & & & 960 & 74 & 100,0 \\
\hline
\end{tabular}




\begin{tabular}{|c|c|c|c|c|c|c|}
\hline $\begin{array}{l}\text { de LAUSANNE } \\
\text { à GENEVE }\end{array}$ & $\begin{array}{l}\text { train } \\
(\text { min. })\end{array}$ & $\begin{array}{l}\text { route } \\
\text { (min.) }\end{array}$ & $\begin{array}{l}\text { rel. de } \\
\text { durée }\end{array}$ & $\begin{array}{l}\text { pendulaires } \\
\text { (chiff. absolus) }\end{array}$ & $\begin{array}{l}\text { \% transports } \\
\text { publics }\end{array}$ & $\begin{array}{l}\text { part du flux } \\
\text { total }\end{array}$ \\
\hline ville/ville 70 & 74 & 63 & 1,17 & 508 & 81 & 44,3 \\
\hline ville/ville 80 & 73 & 63 & 1,16 & 642 & 83 & 34,6 \\
\hline ville/ville 90 & 73 & 63 & 1,16 & 1143 & 86 & 25,5 \\
\hline ville/ville 00 & 74 & 63 & 1,17 & 1367 & 85 & 20,3 \\
\hline ville/couronne 70 & 83 & 63 & 1,32 & 129 & 49 & 11,3 \\
\hline ville/couronne 80 & 82 & 63 & 1,30 & 192 & 46 & 10,3 \\
\hline ville/couronne 90 & 82 & 60 & 1,37 & 612 & 56 & 13,6 \\
\hline ville/couronne 00 & 81 & 57 & 1,42 & 1084 & 52 & 16,1 \\
\hline couronne/ville 70 & 97 & 63 & 1,54 & 372 & 65 & 32,5 \\
\hline couronne/ville 80 & 82 & 63 & 1,30 & 693 & 61 & 37,3 \\
\hline couronne/ville 90 & 81 & 63 & 1,29 & 1493 & 68 & 33,3 \\
\hline couronne/ville 00 & 82 & 63 & 1,30 & 1903 & 66 & 28,3 \\
\hline couronne/couronne 70 & 106 & 63 & 1,68 & 137 & 36 & 12,0 \\
\hline couronne/couronne 80 & 91 & 63 & 1,44 & 330 & 36 & 17,8 \\
\hline couronne/couronne 90 & 90 & 60 & 1,50 & 1241 & 34 & 27,6 \\
\hline couronne/couronne 00 & 89 & 57 & 1,56 & 2442 & 25 & 36,3 \\
\hline agglo/agglo 70 & & & & 1146 & 76 & 100,0 \\
\hline agglo/agglo 80 & & & & 1857 & 63 & 100,0 \\
\hline agglo/agglo 90 & & & & 4489 & 62 & 100,0 \\
\hline agglo/agglo 00 & & & & 6736 & 52 & 100,0 \\
\hline
\end{tabular}

\begin{tabular}{|c|c|c|c|c|c|c|}
\hline $\begin{array}{l}\text { de GENEVE } \\
\text { à LAUSANNE }\end{array}$ & $\begin{array}{l}\text { train } \\
(\text { min. })\end{array}$ & $\begin{array}{l}\text { route } \\
\text { (min.) }\end{array}$ & $\begin{array}{l}\text { rel. de } \\
\text { durée }\end{array}$ & $\begin{array}{l}\text { pendulaires } \\
\text { (chiff. absolus) }\end{array}$ & $\begin{array}{l}\text { \% transports } \\
\text { publics }\end{array}$ & $\begin{array}{l}\text { part du flux } \\
\text { total }\end{array}$ \\
\hline ville/ville 70 & 73 & 63 & 1,16 & 139 & 81 & 25,3 \\
\hline ville/ville 80 & 72 & 63 & 1,14 & 182 & 75 & 18,3 \\
\hline ville/ville 90 & 72 & 60 & 1,20 & 338 & 74 & 16,9 \\
\hline ville/ville 00 & 73 & 60 & 1,22 & 639 & 80 & 15,4 \\
\hline ville/couronne 70 & 99 & 62 & 1,60 & 39 & 23 & 7,1 \\
\hline ville/couronne 80 & 84 & 62 & 1,35 & 72 & 53 & 7,2 \\
\hline ville/couronne 90 & 83 & 59 & 1,41 & 153 & 43 & 7,7 \\
\hline ville/couronne 00 & 84 & 59 & 1,42 & 329 & 42 & 7,9 \\
\hline couronne/ville 70 & 85 & 64 & 1,33 & 267 & 66 & 48,6 \\
\hline couronne/ville 80 & 83 & 64 & 1,30 & 502 & 71 & 50,5 \\
\hline couronne/ville 90 & 83 & 61 & 1,36 & 811 & 65 & 40,6 \\
\hline couronne/ville 00 & 82 & 59 & 1,39 & 1670 & 44 & 40,2 \\
\hline couronne/couronne 70 & 11 & 63 & 0,17 & 104 & 44 & 18,9 \\
\hline couronne/couronne 80 & 95 & 63 & 1,51 & 238 & 32 & 23,9 \\
\hline couronne/couronne 90 & 94 & 60 & 1,57 & 694 & 20 & 34,8 \\
\hline couronne/couronne 00 & 93 & 57 & 1,63 & 1512 & 18 & 36,4 \\
\hline agglo/agglo 70 & & & & 549 & 59 & 100,0 \\
\hline agglo/agglo 80 & & & & 994 & 51 & 100,0 \\
\hline agglo/agglo 90 & & & & 1996 & 45 & 100,0 \\
\hline agglo/agglo 00 & & & & 4150 & 40 & 100,0 \\
\hline
\end{tabular}

Tableaux 2a-d: Durée et relation de durée des trajets, nombre de pendulaires et répartition modale, I 970-2000. 
Fig. ra et rb: Relation entre les pendulaires en transports publics et ceux en voiture $197^{\circ}$ (à gauche) et 2000 (à droite).
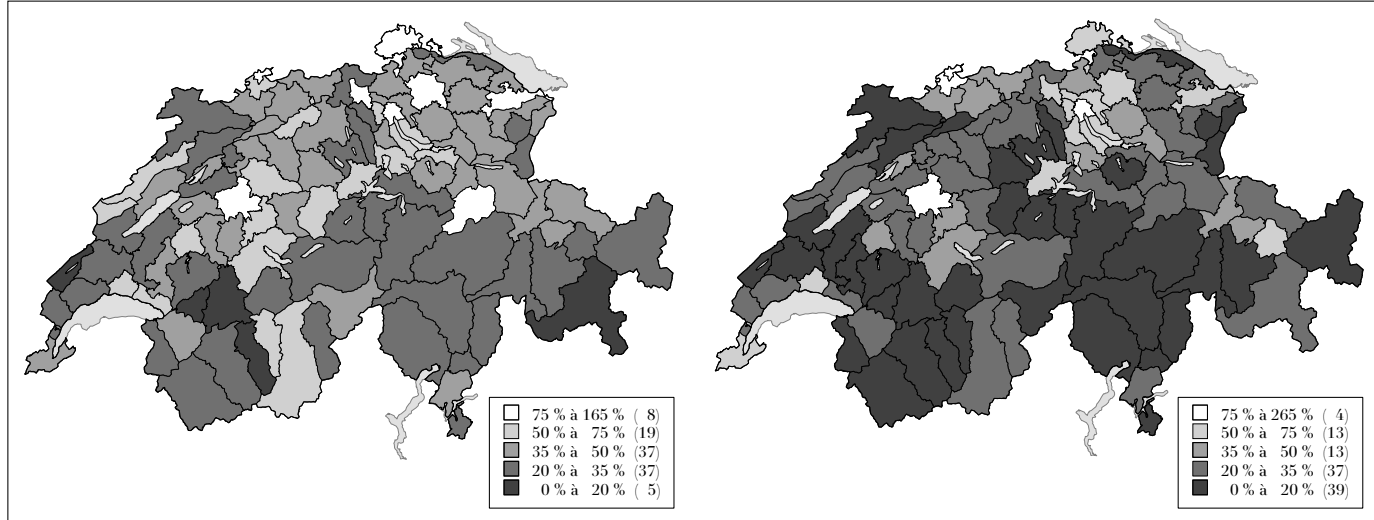

Si au cours des années r 970, la relation de durée des trajets par rail et par route a été favorable à la voiture grâce à l'ouverture de l'autoroute, les années I 98 o et I $99^{\circ}$ ont été propice au train, d'abord sur le tronçon inter-ville principal, puis pour les réseaux urbains terminaux. Cette situation connaîtra une nouvelle amélioration sensible à partir de 2005 avec un gain supplémentaire de onze minutes.

La pendularité à longue distance a donc connu, pour la relation Berne-Zurich, un changement en faveur du train grâce à l'amélioration du part de marché de tous les quatre types de relation et spécialement grâce au renforcement de la relation ville-ville. Cette dernière reflète le gain d'un nouveau marché de pendulaires pour lesquels un trajet d'un peu plus qu'une heure est considéré acceptable, voire concurrentiel par rapport à d'autres types de trajets, plus courts, mais moins propices à l'utilisation du temps de trajet pour déployer d'autres activités.

\subsubsection{Les relations Genève-Lausanne (et vice versa)}

A une distance de moitié plus courte que celle entre Berne et Zurich, la relation Genève-Lausanne est d'une autre nature et permet de considérer la voiture comme une alternative valable au train, d'autant plus que les temps de trajet sur l'autoroute lui sont favorables. En outre, le temps de parcours en train est resté identique depuis plus de 30 ans (34 minutes), tandis que les contournements d'autoroutes de Lausanne (en r 974) et de Genève (en r 993) ont amélioré l'accès aux couronnes situées au-delà de la ville voisine. L'horaire de 2005 prévoit un gain d'une minute sur le tronçon entre les deux villes et une amélioration de la fréquence et de sa distribution horaire.

Sur l'ensemble de flux entre les deux agglomérations, le train n'a cessé de perdre du terrain face à la route: de Lausanne à Genève de $76 \%$ $(\mathbf{r} 970)$ à $63 \%$ (I 980$), 62 \%($ I 990$)$ à $52 \%$ (2000). Dans le sens opposé, de Genève à Lausanne, le recul s'est avéré tout aussi fort et la part modale actuelle du train est encore moins favorable aux transports publics: de $59 \%$ en I 970 à 5 I $\%$, $45 \%$ et à $40 \%$ actuellement.

Bien que les transports publics restent dominants pour la relation entre les deux villescentres et que cette part ait encore augmenté depuis r $99^{\circ}$, ces flux sont devenus très minoritaires (recul de 44\% à $20 \%$ entre Lausanne et Genève et de $25 \%$ à r $5 \%$ dans le sens inverse). Pour toutes les autres relations, le train n'est que très peu fréquenté et en diminution constante (par exemple: i $8 \%$ de la couronne genevoise vers la couronne lausannoise). Ainsi, le recul du train ne peut guère être imputé à des changements de pratiques modales pour des relations origine-destination identiques, mais il est essentiellement le résultat d'une dynamique spatiale de transfert de la croissance de l'habitat et de l'emploi des villes-centres vers leurs couronnes. Entre les deux pôles lémaniques, contrairement à la relation entre Berne et Zurich, les flux destinés aux couronnes accusent un renforcement net. Ces flux sont d'ailleurs en partie de relativement courte distance (par exemple entre Nyon et Morges). Leur majorité se dirige vers les nouvelles zones d'emploi dans l'Ouest ou le Nord lausannois, ou dans la région de l'aéroport de Cointrin, de Vernier ou de Genève Sud (La Praille, Plan-les-Ouates). $75 \%$ à $80 \%$ de ces trajets se font dorénavant en voiture.

\subsection{L'attribution des différences. un effet du contexte culturel ou des distances-temps?}

On pourrait être tenté d'attribuer les grandes différences dans l'utilisation du train que l'on 
observe entre les deux paires d'agglomération alémanique et lémanique au contexte culturel. Pourtant, des explications structurelles liées aux réseaux de transport doivent être évoquées. Une simple comparaison peut soutenir la thèse de l'effet de distance: si $73 \%$ des actifs de l'agglomération de Zurich qui se rendent quotidiennement à Berne utilisent le train, ce pourcentage s'élève à $72 \%$ pour les Bâlois et à $70 \%$ des Lausannois travaillant dans l'agglomération de la capitale - à distance plus ou moins égale, un comportement comparable. Par contre, la liaison Bâle-Zurich, nettement plus courte, est moins orientée sur le train: $43 \%$ des Zurichois se rendant à Bâle, mais $63 \%$ des Bâlois travaillant à Zurich, utilisent le train. En conclusion, le facteur de distance semble donc dominer le facteur culturel.

\section{Synthèse: les transports publics en péril?}

Le recensement de la population est la source la plus riche en matière de données de flux spatialisés désagrégés. Par contre, ces flux ne couvrent que les déplacements quotidiens entre le domicile et le lieu de travail ou le lieu de formation. Les recensements harmonisés de I 970 à 2000 montrent qu'au cours de trois dernières décennies les trajets pendulaires se développent sur des distances toujours croissantes, à une vitesse moyenne toujours plus grande et sur des trajets davantage orientés vers les grandes agglomérations en général et leurs couronnes en particulier. La part des modes lents (et la part des actifs qui travaillent à l'adresse de leur logement) a constamment diminué, au profit des trajets en voiture et en transports publics. La relation entre ces deux modes de transports rapides s'est développée en faveur des transports publics entre 1980 et r $99^{\circ}$ et en faveur de la route dans les années I 970/80 et I990/200o. La dernière décennie a connu un net recul des transports publics urbains, tandis que le train a pu augmenter sa part du marché de $9,8 \%$ à I I, $0 \%$. Cette augmentation est due aux trajets longs entre agglomérations et aux mouvements de pendulaires dans l'aire métropolitaine de Zurich, où le réseau ferroviaire a connu une nette amélioration de l'offre et des temps de parcours. Partout ailleurs, l'utilisation du train stagne ou recule.

L'évolution de la pendularité à longue distance peut être observée le plus facilement entre les grandes agglomérations. Notre analyse montre que ce segment de la pendularité est en très forte croissance, puisqu'un doublement en chiffres absolus des pendulaires s'est opéré

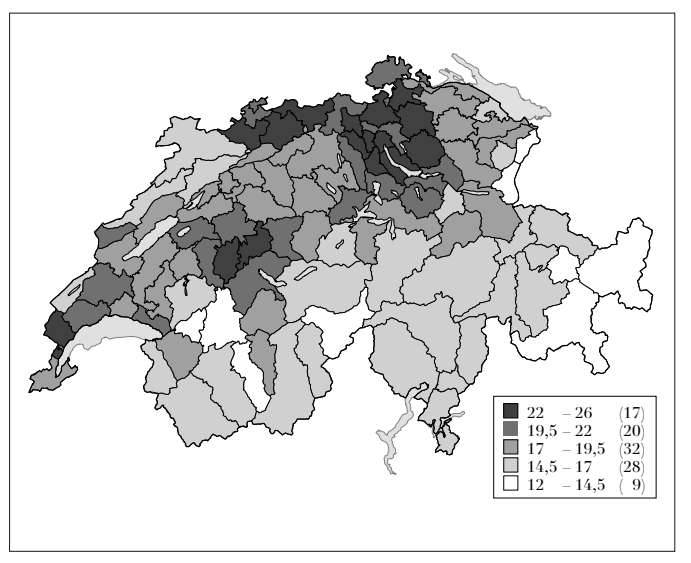

$\operatorname{dis} P_{\text {I }} 6$ I $\cdot 2 / 2005$

dans chaque période intercensitaire. Entre $197^{\circ}$ et r $99^{\circ}$, ces flux inter-agglomération - à l'origine fortement orientés sur le train - se sont partiellement reportés sur la voiture, surtout entre agglomérations séparées d'une distance moyenne, étant donné la part toujours plus importante des origines et destinations dans les couronnes. Depuis r $99^{\circ}$, cette tendance s'est inversée entre Berne et Zurich, grâce à une forte hausse des trajets entre les villes-centres. La concentration continue des emplois spécialisés dans ces villes-centre, la réalisation des premières étapes du programme “Rail+Bus 2000” pour le transport interurbain et l'accessibilité améliorée offertes par les transports urbains (SBahn en particulier) sont les facteurs principaux qui expliquent cette tendance. La qualité du temps de trajet offerte pour les déplacements entre agglomérations a favorisé la croissance de ce type de trajets: à ratio de temps comparable entre voiture et train, les analyses diachroniques des recensements indiquent que l'on prend désormais davantage le train.

La pendularité de longue distance est donc atypique par rapport au constat général d'une persistance de trajets pendulaires de durée moyenne et orientée de plus en plus sur la voiture. De facto, ses adeptes sont des pendulaires de statut socio-professionnel élevé. Leur apport pour une utilisation de transports plus écologiques n'en est pas forcément convaincant, au vu des longues distances qu'ils parcourent. Quant à leur contribution à l'utilisation des transports publics, même si elle est réelle et constitue certainement une niche de marché importante pour les CFF, elle ne peut cacher un recul massif de l'usage des transports en commun en général pour aller travailler.

$\mathrm{Au}$ cours des trente dernières années, les villes-centre des agglomérations suisses ont pu maintenir une forte concentration des emplois,
Fig. 2: Durée moyenne d'un trajet pendulaire en minutes, 2000 (actifs ayant un trajet). 
Prof. Martin Schuler

Ecole polytechnique fédérale de Lausanne

Laboratoire Chôros

Station i 6

CH-го 5 Lausanne

martin.schuler@epfl.ch

Prof. Vincent Kaufmann Ecole polytechnique fédérale de Lausanne

Laboratoire de Sociologie

Urbaine

Station 16

CH-ı I 5 Lausanne

vincent.kaufmann@epfl.ch malgré la création de pôles de développement dans des banlieues bien desservies par les autoroutes. Cette situation a certainement profité des grands investissements dans les systèmes de transport urbains. Par contre, elle ne s'explique pas par une politique active de limitation des l'accès des centre-villes à la voiture (si ce n'est le périmètre des vieilles villes historiques). La discussion récente du péage urbain aurait, selon notre analyse, un effet positif au flux à longue distance en train vers les villes centre et, par conséquent, un impact supplémentaire vers une spécialisation encore plus forte des emplois à haute qualification dans au cœur des agglomérations.

En fin de compte, le recensement de la population de 2000 met en avant que les efforts entrepris par les collectivités publiques en faveur d'une promotion des transports publics n'ont trouvé que peu de résonance. Ceci met le doigt une fois encore sur la nécessité d'accompagner l'amélioration des offres de transports en commun d'autres mesures. A quoi sert-il d'avoir une offre de qualité si l'urbanisation se fait en dehors des périmètres qu'elle dessert? A quoi sertil de développer des transports publics efficaces si cette politique n'est pas accompagnée d'une gestion cohérente du stationnement? Les résultats obtenus à Zurich sont fondamentalement le fruit d'une action coordonnée entre développement des transports publics gestion des accessibilités routières et localisation de la croissance des activités. Quand la Suisse romande va-t-elle enfin l'entendre?

\section{Notes}

Les auteurs tiennent à remercier le Prof. AndréGilles Dumont et son équipe du LAVOC - EPFL, ainsi M. Daniel Emery du LITEP - EPFL pour les échanges scientifiques et documentaires en lien avec cet article.

I Les quatre derniers recensements de la population suisse permettent un traitement quasiment identique du sujet de la pendularité, puisque la formulation de la question n'a guère changé (en ce qui concerne la saisie des moyens de transport, mais également la durée des trajets) et le traitement des données est resté comparable. Il est vrai que le recensement a quelques difficultés pour attribuer correctement les trajets quotidiens à longue distance, puisque de nombreuses personnes dans cette situation disposent d'un double domicile (et que donc la pendularité n'est pas quotidienne, mais hebdomadaire). La saisie du double domicile n'a été introduite que lors du recensement de I990; depuis les effectifs sont plus fiables, mais entaché par le fait que la part des personnes actives sans indication du lieu de travail a auggmenté.
2 Les calculs des trajets à l'intérieur des agglomérations respectives ont été réalisés à l'aide de cinq lieux représentatifs dans la ville centre et dans l'agglomération. Pour les quatre années observées, l'introduction de nouvelles offres de transport a été prise en compte, ainsi que des changements de des durées des trajets. Par contre, nous n'avons pas pris en considération les effets de congestion.

\section{Bibliographie}

ARE (200I): La mobilité en Suisse - Résultats du microrecensement 2000 sur le comportement de la population en matière de transports. DFI-DETEC/ ARE-OFS, Bern et Neuchâtel.

Bernath, H.-J.; Schlegel, W. et Germann, A. (1994): Kapazitätsgrenzen des Schweizerischen Nationalstrassennetzes. Forschungsauftrag 62/94 auf Antrag des Bundesamtes für Strassenbau, Zürich.

Bürgi-Schmelz, A.; Haug, W.; Schuler, M. et WegeLIN, F. (2003): Pendularité et nouvelle définition des agglomérations. Conférence de presse du I 5 mai 2003 , Office fédéral de la statistique.

CFF: divers horaires historiques, ainsi que le site www.cff.ch.

Crozet, Y. et Joly, I. (2003): De l'hypothèse de la constance du budget temps de transport à sa remise en cause: Une double interpellation des politiques de transport urbain. Laboratoire d'Economie des Transports, ENTPE, Lyon 2.

Flamm, M. (2004), Comprendre le choix modal - les déterminants des pratiques modales et des représentations individuelles des moyens de transport. Thèse $\mathrm{n}^{\circ}{ }_{2} 897$, EPFL, Lausanne.

Frick, R.; Wüthrich, P.; Zbinden, R. et Keller, M. (2004): Pendlermobilität in der Schweiz. Übersichtsanalyse Volkszählung 20oo. Bundesamt für Statistik und Bundesamt für Raumplanung, Neuchâtel et Berne.

Jemelin, Ch.; Kaufmann, V. et Barbey, J. (2003): Inégalités d'accès et politique des transports urbains: une comparaison internationale. Rapport intermédiaire à l'intention du PREDIT.

Kaufmann, V.; Jemelin, Сh. et Joye, D. (2000): Les gares-interfaces entre ruptures et activités. Rapport du PNR 4i A4, Berne.

LÉvy, J. (2000): “Les nouveaux espaces de la mobilité». In: Bonnet M. et Desjeux D. (éd.): Les territoires de la mobilité. PUF, Paris.

Office fédéral de routes: Info, Autoroutes suisses. annuel.

Schuler, M. et Kaufmann, V. (1996): “Pendularité à longue distance, la vitesse des transports comme facteur structurant de l'urbain». En: DISP n ${ }^{\circ}$ г 6, Zurich, 3-го.

Schuler, M.; Perlik, M. et Pasche, N. (2004): Nonurbain, campagne ou périphérie - où se trouve l'espace rural aujourd'hui? Office fédéral du développement territorial, Berne.

Viard, J. (I995): La société d'archipels. Editions de l'Aube, La Tour d'Aigues. 